\title{
CORRIGENDUM: siRNA-directed inhibition of HIV-1 infection
}

Carl D Novina, Michael F Murray, Derek M Dykxhoorn, Paul J Beresford, Jonathan Riess, Sang-Kyung Lee, Ronald G Collman, Judy Lieberman, Premlata Shankar \& Phillip A Sharp

Nat. Med. 8, 681-686 (2002)

The reported antisense strand of the CD19 siRNA used as a negative control in Figure 3 (p. 683), with the sequence 5'-GAAUCAUCCUCCGUCCGGGGU-3', did not have the appropriate sequence. The CD19 RNA used in Figure 3 is therefore not relevant as an unrelated siRNA control. Additional experiments using other control siRNAs confirmed that the silencing phenomenon reported in Figure 3 is specific; the conclusions of the paper remain unchanged. The authors regret the error.

ERRATUM: TNF defined as a therapeutic target for rheumatoid arthritis and other autoimmune diseases

Marc Feldmann \& Ravinder N Maini

Nat. Med. 9, 1245-1250 (2003)

A callout for Figure 3 should have been inserted on p. 1248. The last sentence of the first paragraph of column 3 should read, “...the relative rarity of sustained remission of disease (Fig. 3).” We regret the error.

\section{ERRATUM: $\beta$-receptor polymorphisms: heart failure's crystal ball}

David A Kass

Nat. Med. 9, 1260-1262 (2003)

The last sentence of the Figure 1 legend (p. 1261) is incomplete. The sentence should read, “...contractile depression compared with Gly389 hearts. MHC, myosin heavy chain." We regret the error.

\section{ERRATUM: Confronting ancient scourges (cover image)}

Nat. Med. 9 (2003)

The cover image for the May 2003 issue was not credited. The last sentence of the cover caption should read, "Courtesy of S. Kaufmann and J. Golecki/SPL/Photo Researchers Inc." We regret the error. 